\title{
Establishing Classifiers With Clinical Laboratory Indicators to Distinguish COVID-19 From Community-Acquired Pneumonia: Retrospective Cohort Study
}

Wanfa Dai $^{1 *}$, MD; Pei-Feng Ke ${ }^{2,3 *}$, MPH; Zhen-Zhen Li ${ }^{4}$, BSc; Qi-Zhen Zhuang ${ }^{4}$, BSc; Wei Huang ${ }^{1}$, MD; Yi Wang ${ }^{2,4}$, MPhil; Yujuan Xiong ${ }^{2,3 *}, \mathrm{PhD}$; Xian-Zhang Huang ${ }^{2,3 *}, \mathrm{PhD}$

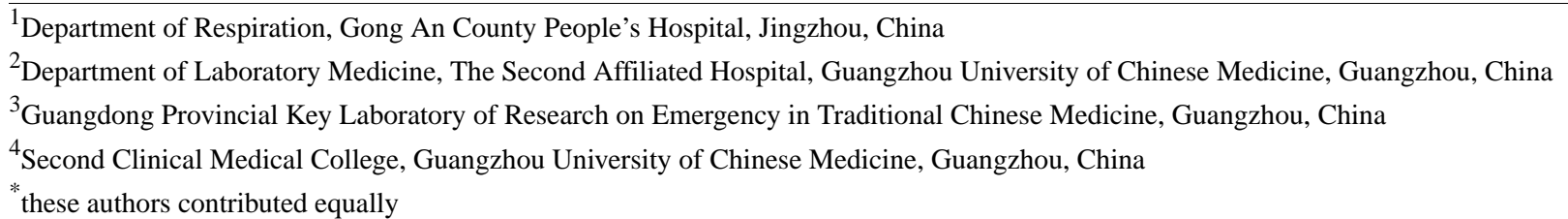

\section{Corresponding Author:}

Xian-Zhang Huang, PhD

Department of Laboratory Medicine

The Second Affiliated Hospital

Guangzhou University of Chinese Medicine

111 Dade Rd

Guangzhou, 510210

China

Phone: 8602081887233 ext 35362

Email: huangxz020@gzucm.edu.cn

\section{Abstract}

Background: The initial symptoms of patients with COVID-19 are very much like those of patients with community-acquired pneumonia (CAP); it is difficult to distinguish COVID-19 from CAP with clinical symptoms and imaging examination.

Objective: The objective of our study was to construct an effective model for the early identification of COVID-19 that would also distinguish it from CAP.

Methods: The clinical laboratory indicators (CLIs) of 61 COVID-19 patients and 60 CAP patients were analyzed retrospectively. Random combinations of various CLIs (ie, CLI combinations) were utilized to establish COVID-19 versus CAP classifiers with machine learning algorithms, including random forest classifier (RFC), logistic regression classifier, and gradient boosting classifier (GBC). The performance of the classifiers was assessed by calculating the area under the receiver operating characteristic curve (AUROC) and recall rate in COVID-19 prediction using the test data set.

Results: The classifiers that were constructed with three algorithms from 43 CLI combinations showed high performance (recall rate $>0.9$ and AUROC $>0.85$ ) in COVID-19 prediction for the test data set. Among the high-performance classifiers, several CLIs showed a high usage rate; these included procalcitonin (PCT), mean corpuscular hemoglobin concentration (MCHC), uric acid, albumin, albumin to globulin ratio (AGR), neutrophil count, red blood cell (RBC) count, monocyte count, basophil count, and white blood cell (WBC) count. They also had high feature importance except for basophil count. The feature combination (FC) of PCT, AGR, uric acid, WBC count, neutrophil count, basophil count, RBC count, and MCHC was the representative one among the nine FCs used to construct the classifiers with an AUROC equal to 1.0 when using the RFC or GBC algorithms. Replacing any CLI in these FCs would lead to a significant reduction in the performance of the classifiers that were built with them.

Conclusions: The classifiers constructed with only a few specific CLIs could efficiently distinguish COVID-19 from CAP, which could help clinicians perform early isolation and centralized management of COVID-19 patients.

(J Med Internet Res 2021;23(2):e23390) doi: 10.2196/23390

\section{KEYWORDS}

COVID-19; clinical laboratory indicators; community-acquired pneumonia; classifier; classification algorithm 


\section{Introduction}

COVID-19 caused by SARS-CoV-2 infection, which was discovered in early December 2019, has become a global pandemic. As of August 3, 2020, COVID-19 has become widespread in 215 countries, areas, or territories worldwide; it has caused infection in more than 17.9 million people and has resulted in the deaths of more than 686,000 people [1]. The World Health Organization has stated that the spread of COVID-19 may be impeded by early detection, isolation, and the implementation of a robust health care system [2,3]. Nevertheless, the published data indicate that the initial symptoms of COVID-19 in patients are very similar to those in patients with the common cold or influenza. COVID-19 patients exhibit different clinical symptoms, and some of them do not have any symptoms [4-7]. sars-cov-2 infection has a long incubation period, with a median incubation period of 5 to 7 days, which is the chief risk factor for community infection [6,8]. Community-acquired pneumonia (CAP) and COVID-19 have similar clinical and imaging features, but their treatment and infectivity are very dissimilar. Distinguishing COVID-19 from CAP is very important to prevent the spread of COVID-19 and to provide specific treatment.

Some characteristic spectra demonstrated by clinical laboratory indicators (CLIs) of COVID-19 patients have been utilized as auxiliary clues for diagnosis [9]. Previous studies have demonstrated that increased procalcitonin (PCT), lymphocytopenia, and thrombin activation can all be utilized as auxiliary diagnostic indicators of COVID-19 and poor prognostic factors [9-11]. However, they are also correlated with CAP [12-15]. Thus, in accordance with the changes in these indicators, it is impossible to differentiate COVID-19 from CAP. The changes in the neutrophil to lymphocyte ratio, the peak platelet to lymphocyte ratio, lactate dehydrogenase (LDH), C-reactive protein (CRP), and interleukin-6 (IL-6) are considered to be associated with the progression and prognosis of COVID-19 [9], but using the information from the CLIs to give clinicians correct guidance is still a great challenge.

Classifiers established by machine learning (ML) algorithms based on various clinical features, biomarkers, and CLIs are increasingly widely utilized in disease diagnosis and risk prediction [16]. During the COVID-19 pandemic, ML was also widely used to predict, classify, assess, track, and control the spread of SARS-CoV-2 [17,18]. ML can improve diagnostic performance compared with hand-selected biomarkers by selecting relevant biomarkers and more consistently capturing both their relative importance to prediction and their interactions among one another [19]. In this study, we used CLIs to build classifiers with different ML algorithms to distinguish COVID-19 patients from CAP patients; we found that only the feature combinations (FCs) with many specific CLIs rather than the FCs with the most significantly differential CLIs between the two groups could build high-performance classifiers (HPCs).

\section{Methods}

\section{Collection of Patients' Electronic Medical Record Data}

The electronic medical records of patients who were admitted to Gong An County People's Hospital, China, and diagnosed with COVID-19 or CAP from December 2019 to March 2020 were retrieved. The information regarding each patient's age, sex, clinical symptoms upon admission, medical history, epidemiological history, computed tomography (CT) imaging features, and CLIs were sorted out for retrospective analysis. Only the laboratory test results during admission were included. It was specified that all patients' data were to be kept confidential, and this data were only to be utilized for comprehensive analysis. No personal information about any patient was mentioned in the paper. This study was approved by the ethics committees from the Guangdong Provincial Hospital of Chinese Medicine (approval No. ZE2020-049-01) with a waiver of informed consent due to the retrospective nature of the study.

\section{Data Description}

Diagnosis and clinical classification of COVID-19 were performed according to the Chinese Clinical Guidance for COVID-19 Pneumonia Diagnosis and Treatment (7th edition) [20]. A total of 61 patients with COVID-19 and 60 patients with CAP were enrolled according to the discharge diagnosis on their electronic medical records. There were 3 mild, 47 common, 6 severe, and 5 critical types, which were categorized into two groups for further analysis as follows: COVID19-COM (3 mild and 47 common types) and COVID19-SV (6 severe and 5 critical types). They were matched by age and sex and did not significantly differ in terms of medical history. The main clinical symptoms between CAP and COVID-19 groups were not significantly different.

\section{Primary Analysis}

The descriptive analysis of all CLIs was performed between groups or subgroups. Between-group or between-subgroup differences were tested using the statsmodels module from Python (Python Software Foundation) [21]. The Student $t$ test was performed when the distribution of the variables conformed to the normal distribution; otherwise, the Mann-Whitney $U$ test was used. The chi-square test was used to detect differences in baseline data between two groups or subgroups. A value of $P<.05$ was considered to be significant.

\section{Feature Selection and Data Preprocessing}

The CLIs with a missing value ratio greater than $20 \%$ were excluded. Only the CLIs with a significant difference between the two groups were selected and used to generate $1,807,780$ nonrepetitive random FCs, consisting of one to eight CLIs, by using the combinations iterator in the itertools module from Python [22]. Next, an FC was selected from the FC list one by one to form a new data sheet with the dependent variable (ie, disease type), and 1,807,780 new data sheets were eventually formed. For each new data sheet, the rows with missing values were removed. The remaining rows were then divided into training_dataset and test_dataset using scikit-learn, version 0.23.1 (train_test_split function with test_size $=0.25$, 
random_state $=0$ ). The training data set was used to build the classifier, and the test data set was used to assess the performance. The feature values were standardized using the StandardScaler function in the scikit-learn module before constructing the logistic regression (LR) classifier.

\section{Construction of Classifiers With ML Algorithms in the Scikit-Learn Module}

Scikit-learn is a Python module integrating a wide range of state-of-the-art ML algorithms for medium-scale supervised and unsupervised problems [23]. The LR classifier, the random forest classifier (RFC), and the gradient boosting classifier (GBC) have been typically used to construct classifiers in prediction of disease risk, progression, prognosis, and so on [24]. The LR classifier in the sklearn.linear_model is also known as logit regression, maximum-entropy classification, or the log-linear classifier. In this model, the probabilities describing the possible outcomes of a single trial are modeled using a logistic function [24]. The RFC in the sklearn.ensemble module is one of the averaging algorithms in ensemble methods and is a perturb-and-combine technique specifically designed for trees. In the random forest algorithm, each tree in the ensemble is built from a sample drawn with replacement from the training data set. Furthermore, when splitting each node during the construction of a tree, the best split is found either from all input features or from a random subset of size setting with the parameter max_features. In practice, the variance reduction due to the introduction of randomness in the classifier construction is often significant, hence, yielding an overall better model $[25,26]$. The GBC algorithm, using the sklearn.ensemble function, is a boosting method, in which base estimators are built sequentially. To reduce the bias of the combined estimator, one has to combine several weak models to produce a powerful ensemble. The GBC algorithm builds an additive model in a forward stage-wise fashion, and it allows for the optimization of arbitrary differentiable loss functions [27,28].

In this study, the classifiers were respectively constructed using the LR classifier, RFC, and GBC in the scikit-learn module with the training data set. The model parameter settings were kept as default, except that random_state was modified to " 0 " for all models and class_weight was modified to "balanced" for the LR classifier and RFC models. The performance of the classifiers was evaluated with the test data set by calculating the recall rate (ie, sensitivity), specificity, accuracy, and area under the receiver operating characteristic curve (AUROC), using the sklearn_metrics.recall_score, sklearn_metrics.precis ion_score, sklearn_metrics.accuracy_score, and sklearn_metrics.auc functions, respectively. Gini importance was computed using the feature_importance function to measure the importance of each feature in the RFC and the GBC. The higher the Gini importance value, the more important the feature [29]. All the above analyses were performed in Python, version 3.7 (Python Software Foundation).

\section{Results}

\section{Basic Characteristics of CAP Group and COVID-19 Group}

No significant differences in age and sex were found between CAP and COVID-19 groups (see Table 1); however, the proportions of males in the CAP and COVID-19 groups were $55 \%(33 / 60)$ and $66 \%$ (40/61), respectively, and were higher than those of females in both groups. No significant difference in the medical history between the two groups (see Table 1) was observed. Also, no significant difference was found in the proportions of the main clinical symptoms between the two groups, such as fever, cough, fatigue, muscle soreness, and loss of appetite (see Table 1). The average hospitalization days for CAP patients were remarkably lower than those for COVID-19 patients $(P<.001)$. In the CAP group, some patients with pulmonary $\mathrm{CT}$ also had imaging features that included patchy hyperdense shadow $(11 / 60,18 \%)$, ground-glass shadow $(4 / 60$, $7 \%)$, and fibrotic lesion $(6 / 60,10 \%)$. Nonetheless, the chief imaging features of pulmonary CT in the COVID-19 group were patchy hyperdense shadow $(25 / 61,41 \%)$ and ground-glass shadow $(9 / 61,15 \%)$, and many patients $(7 / 61,11 \%)$ had both patchy hyperdense shadow and ground-glass shadow (see Table $1)$. Among the 61 patients suffering from COVID-19, $3(5 \%)$ had mild symptoms, 47 (77\%) had common symptoms, $6(10 \%)$ had severe symptoms, and $5(8 \%)$ had critical symptoms. Fever and cough were the principal symptoms in the early stage of COVID-19, and these accounted for 70\% (43/61) and 64\% $(39 / 61)$ of the cases, respectively (see Table 1). Among the CAP patients included in the analysis, no cases of death were found during hospitalization; however, 3 of the $5(60 \%)$ severely ill patients in the COVID-19 group, who were aged 36, 49, and 74 years, died during hospitalization. The 36-year-old patient who died underwent interventricular septal repair in childhood. 
Table 1. Comparison of baseline information between COVID-19 patients and community-acquired pneumonia (CAP) patients.

\begin{tabular}{|c|c|c|c|}
\hline Baseline characteristic & CAP patients $(n=60)$ & COVID-19 patients $(\mathrm{n}=61)$ & $P$ value \\
\hline Sex (male), n (\%) & $33(55)$ & $40(66)$ & .27 \\
\hline Age (years), mean (SD) & $55.72(18.10)$ & $50.23(16.95)$ & .09 \\
\hline Hospitalization days, median (IQR) & $9(7-12)$ & $21(13-26)$ & $<.001$ \\
\hline \multicolumn{4}{|l|}{ Medical history, n (\%) } \\
\hline Hypertension & $14(23)$ & $16(26)$ & .83 \\
\hline Diabetes & $2(3)$ & $6(10)$ & .27 \\
\hline Liver disease & $2(3)$ & $3(5)$ & .99 \\
\hline Heart disease & $3(5)$ & $5(8)$ & .72 \\
\hline Exposure history & Unclear & $54(89)$ & $\mathrm{N} / \mathrm{A}^{\mathrm{a}}$ \\
\hline Familial aggregation infection ${ }^{b}$ & Unclear & $22(36)$ & N/A \\
\hline \multicolumn{4}{|l|}{ Initial symptoms, n (\%) } \\
\hline Fever & $36(60)$ & $43(70)$ & .26 \\
\hline Cough & $44(73)$ & $39(64)$ & .33 \\
\hline Myalgia & $4(7)$ & $7(11)$ & .53 \\
\hline Poor appetite & $5(8)$ & $11(18)$ & .18 \\
\hline Fatigue & $33(55)$ & $24(39)$ & .10 \\
\hline Days from onset of symptoms to admission, median (IQR) & Unrecorded & $3(1-7)$ & N/A \\
\hline \multicolumn{4}{|l|}{ Imaging features, $n(\%)$} \\
\hline Patchy high-density opacity & $11(18)$ & $25(41)$ & .009 \\
\hline Ground-glass opacity & $4(7)$ & $9(15)$ & .24 \\
\hline Fibrotic lesion & $6(10)$ & $3(5)$ & .32 \\
\hline Patchy high-density opacity and ground-glass opacity & $0(0)$ & $7(11)$ & .01 \\
\hline Death cases, $\mathrm{n}(\%)$ & $0(0)$ & $3(5)$ & N/A \\
\hline
\end{tabular}

${ }^{\mathrm{a}} \mathrm{N} / \mathrm{A}$ : not applicable; groups could not be compared because there were no values for the CAP group.

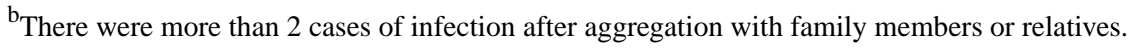

\section{Characteristic Profile of the CLIs in COVID-19 and CAP}

Even though most CLIs had a similar variation trend in both CAP and COVID-19, the extent of change was different. Among more than 60 evaluated CLIs, there were significant differences in 25 CLIs between the two groups (see Table 2). A decrease of lymphocyte, red blood cell (RBC) count, hematocrit or packed-cell volume (PCV), hemoglobin concentration, and mean corpuscular hemoglobin concentration (MCHC) and an increase of neutrophil ratio, prothrombin time (PT), micro-CRP (mCRP), and PCT were observed in both COVID-19 and CAP patients. Furthermore, the neutrophil ratio and levels of PT, mCRP, and PCT in CAP were remarkably higher than those in COVID-19. Levels of lymphocyte, RBC count, PCV, hemoglobin concentration, and $\mathrm{MCHC}$ in $\mathrm{CAP}$ were significantly lower than those in COVID-19 (see Figure 1). Various erythrocyte-related CLIs-RBC count, PCV, hemoglobin concentration, and $\mathrm{MCHC}$ - significantly decreased in both CAP and COVID-19, but there was a greater reduction in CAP patients (see Figure 1). The RBC distribution width-standard deviation (RDW-SD) and RBC mean corpuscular volume (MCV) also indicated prominent differences between CAP and COVID-19 (see Figure 1). 
Table 2. Differences in clinical laboratory indicators (CLIs) between patients with community-acquired pneumonia (CAP) and COVID-19.

\begin{tabular}{|c|c|c|c|c|c|}
\hline \multirow[t]{2}{*}{ CLI } & \multicolumn{2}{|c|}{ CAP patients $(n=60)$} & \multicolumn{2}{|c|}{ COVID-19 patients $(\mathrm{n}=61)$} & \multirow[t]{2}{*}{$P$ value } \\
\hline & $\mathrm{n}(\%)$ & Mean (SD) & $\mathrm{n}(\%)$ & Mean (SD) & \\
\hline Procalcitonin (ng/mL) & $43(72)$ & $0.629(0.838)$ & $55(90)$ & $0.134(0.184)$ & $<.001$ \\
\hline Monoamine oxidase B (U/L) & $35(58)$ & $4.569(1.748)$ & $53(87)$ & $3.538(1.592)$ & .001 \\
\hline Myoglobin (ng/mL) & $14(23)$ & $39.179(29.421)$ & $23(38)$ & $65.794(87.039)$ & .04 \\
\hline Micro-C-reactive protein $(\mathrm{mg} / \mathrm{L})$ & $41(68)$ & $63.943(64.530)$ & $13(21)$ & $22.568(29.577)$ & .004 \\
\hline Prothrombin time (seconds) & $30(50)$ & $12.780(0.873)$ & $53(87)$ & $12.460(1.107)$ & .04 \\
\hline Thrombin time (seconds) & $30(50)$ & $15.123(1.565)$ & $53(87)$ & $14.655(1.422)$ & .049 \\
\hline Albumin $(\mathrm{g} / \mathrm{L})$ & $53(88)$ & $35.508(5.929)$ & $54(89)$ & $37.831(6.169)$ & .04 \\
\hline Albumin to globulin ratio & $53(88)$ & $1.211(0.295)$ & $54(89)$ & $1.378(0.482)$ & .047 \\
\hline$\alpha$-L-fucosidase (U/L) & $35(58)$ & $17.709(5.167)$ & $50(82)$ & $22.106(5.698)$ & $<.001$ \\
\hline Uric acid $(\mu \mathrm{mol} / \mathrm{L})$ & $44(73)$ & $284.193(118.608)$ & $54(89)$ & $325.261(92.914)$ & .007 \\
\hline Potassium (mmol/L) & $54(90)$ & $3.900(0.462)$ & $55(90)$ & $4.021(0.392)$ & .03 \\
\hline White blood count cell $\left(\times 10^{9} / \mathrm{L}\right)$ & $58(97)$ & $8.858(5.576)$ & $56(92)$ & $5.293(2.047)$ & $<.001$ \\
\hline Neutrophils (\%) & $57(95)$ & $72.958(15.544)$ & $56(92)$ & $66.661(14.013)$ & .007 \\
\hline Lymphocytes (\%) & $56(93)$ & $18.646(13.416)$ & $56(92)$ & $24.014(11.175)$ & .002 \\
\hline Neutrophil count $\left(\times 10^{9} / \mathrm{L}\right)$ & $56(93)$ & $6.797(5.525)$ & $56(92)$ & $3.649(1.949)$ & $<.001$ \\
\hline Monocyte count $\left(\times 10^{9} / \mathrm{L}\right)$ & $55(92)$ & $0.565(0.337)$ & $56(92)$ & $0.404(0.194)$ & .009 \\
\hline Eosinophil count $\left(\times 10^{9} / \mathrm{L}\right)$ & $55(92)$ & $0.111(0.213)$ & $56(92)$ & $0.053(0.072)$ & .03 \\
\hline Basophil count $\left(\times 10^{9} / \mathrm{L}\right)$ & $55(92)$ & $0.021(0.013)$ & $56(92)$ & $0.015(0.013)$ & .002 \\
\hline Red blood cell count $\left(\times 10^{12} / \mathrm{L}\right)$ & $56(93)$ & $4.028(0.647)$ & $56(92)$ & $4.284(0.570)$ & .008 \\
\hline Hemoglobin concentration $(\mathrm{g} / \mathrm{L})$ & $55(92)$ & $120.800(17.326)$ & $56(92)$ & $130.143(16.888)$ & .005 \\
\hline Packed-cell volume (hematocrit) (L/L) & $55(92)$ & $0.371(0.052)$ & $56(92)$ & $0.389(0.049)$ & .04 \\
\hline Mean red blood cell volume (fL) & $55(92)$ & $93.255(6.662)$ & $56(92)$ & $91.241(6.501)$ & .01 \\
\hline Mean corpuscular hemoglobin concentration (g/L) & $55(92)$ & $325.473(8.360)$ & $56(92)$ & $334.482(13.559)$ & $<.001$ \\
\hline Red blood cell distribution width-standard deviation (fL) & $55(92)$ & $41.476(2.573)$ & $56(92)$ & $41.141(4.082)$ & .01 \\
\hline
\end{tabular}


Figure 1. The statistical distribution of the plasma level of the clinical laboratory indicators (CLIs) with a remarkable difference between COVID-19 and community-acquired pneumonia (CAP). The statistical distribution was presented with a box and whisker plot. The horizontal lines within the boxes indicate the median value. The vertical lines extending below and above the boxes represent 5\%-95\% percentile values. The scale on the y-axis represents the values of the 5th, 25th, 50th, 75th, and 95th percentiles of the CLI in the CAP group. The triangles represent the upper and lower limits of the normal reference range of the laboratory index. AFU: $\alpha$-L-fucosidase; AGR: albumin to globulin ratio; ALB: albumin; BASOC: basophil count; EOC: eosinophil count; HGB: hemoglobin concentration; K: potassium; LYM: lymphocyte; MAO-B: monoaminoxidase B; MCHC: mean corpuscular hemoglobin concentration; mCRP: micro-C-reactive protein; MCV: mean (red blood cell) corpuscular volume; MOC: monocyte count; NEUT: neutrophil ratio; NEUTC: neutrophil count; PCT: procalcitonin; PCV: packed-cell volume (hematocrit); PT: prothrombin time; RBC: red blood cell count; RDW-SD: red blood cell distribution width-standard deviation; TT: thrombin time; UA: uric acid; WBC: white blood cell count.
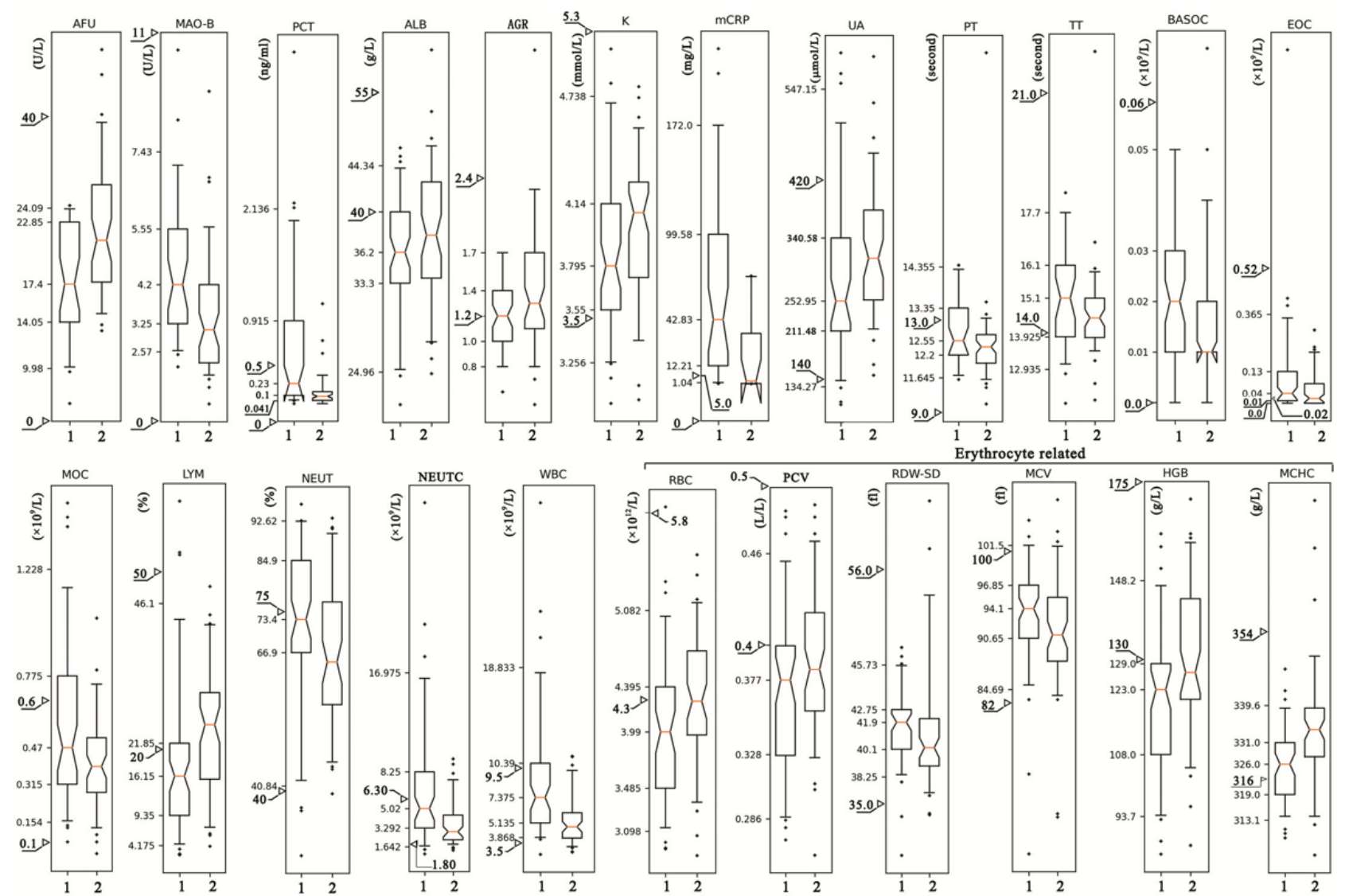

Groups: 1, Patients with Community-Acquired Pneumonia; 2, COVID-19 Patients

Comparing the COVID19-COM and COVID19-SV subgroups, 26 CLIs demonstrated a remarkable difference (see Table 3). In comparison with the COVID19-COM subgroup, LDH, aspartate aminotransferase, fibrinogen content, mCRP, and erythrocyte sedimentation rate increased acutely in the COVID19-SV subgroup, whereas prealbumin, carbon dioxide binding capacity, lymphocytes, and lymphocyte count decreased in the COVID19-SV subgroup (see Multimedia Appendix 1).
An orderly increase of $\alpha$-L-fucosidase (AFU), myoglobin, uric acid, and $\mathrm{MCHC}$ and an orderly decrease of thrombin time, monocyte count, eosinophil count, RBC MCV, and RDW-SD were observed in CAP, COVID19-COM, and COVID19-SV patients, indicating that these CLIs may be used to distinguish CAP from COVID-19 and may suggest the probability of severe COVID-19 progression (see Multimedia Appendix 2). 
Table 3. Difference in clinical laboratory indicators (CLIs) between patients with common and severe types of COVID-19.

\begin{tabular}{|c|c|c|c|c|c|}
\hline \multirow[t]{2}{*}{ CLIs } & \multicolumn{2}{|c|}{$\begin{array}{l}\text { Patients with a common type of COVID- } \\
19(n=50)\end{array}$} & \multicolumn{2}{|c|}{$\begin{array}{l}\text { Patients with a severe type of COVID- } \\
19(n=11)\end{array}$} & \multirow[t]{2}{*}{$P$ value } \\
\hline & $\mathrm{n}(\%)$ & Mean (SD) & $\mathrm{n}(\%)$ & Mean (SD) & \\
\hline Procalcitonin $(\mathrm{ng} / \mathrm{mL})$ & $44(88)$ & $0.112(0.170)$ & $11(100)$ & $0.224(0.217)$ & .01 \\
\hline N-terminal pro-B-type natriuretic peptide (pg/mL) & $29(58)$ & $366.053(549.429)$ & $11(100)$ & $534.782(398.067)$ & .03 \\
\hline Hypersensitive C-reactive protein (mg/L) & $41(82)$ & $23.332(34.483)$ & $11(100)$ & $72.458(60.805)$ & .002 \\
\hline Lactate dehydrogenase (U/L) & $26(52)$ & $214.896(73.319)$ & $8(73)$ & $314.750(118.755)$ & .02 \\
\hline D-dimer (mg/L) & $42(84)$ & $0.834(1.115)$ & $11(100)$ & $5.133(10.399)$ & .005 \\
\hline Myoglobin (ng/mL) & $16(32)$ & $49.221(60.505)$ & $7(64)$ & $103.674(127.354)$ & .02 \\
\hline Cardiac troponin $(\mathrm{ng} / \mathrm{mL})$ & $16(32)$ & $0.011(0.003)$ & $7(64)$ & $0.033(0.041)$ & .02 \\
\hline Creatine kinase (U/L) & $27(54)$ & $81.296(47.153)$ & $8(73)$ & $202.125(195.052)$ & .02 \\
\hline Fibrinogen content (mg/dL) & $42(84)$ & $411.905(104.363)$ & $11(100)$ & $467.455(76.500)$ & .03 \\
\hline Aspartate aminotransferase (U/L) & $46(92)$ & $29.413(15.756)$ & $10(91)$ & $45.600(18.969)$ & .004 \\
\hline$\gamma$-glutamyl transpeptidase (U/L) & $44(88)$ & $46.046(41.609)$ & $10(91)$ & $80.000(44.229)$ & .007 \\
\hline Albumin $(g / L)$ & $44(88)$ & $38.602(6.267)$ & $10(91)$ & $34.440(4.558)$ & .02 \\
\hline Albumin to globulin ratio & $44(88)$ & $1.436(0.507)$ & $10(91)$ & $1.120(0.230)$ & .02 \\
\hline Indirect bilirubin $(\mu \mathrm{mol} / \mathrm{L})$ & $44(88)$ & $9.482(3.841)$ & $10(91)$ & $7.960(4.336)$ & .048 \\
\hline Prealbumin (mg/L) & $41(82)$ & $180.171(83.374)$ & $9(82)$ & $125.556(68.182)$ & .03 \\
\hline$\beta 2$-microglobulin (mg/L) & $41(82)$ & $1.978(0.430)$ & $9(82)$ & $2.528(1.015)$ & .01 \\
\hline Carbon dioxide binding capacity $(\mathrm{mmol} / \mathrm{L})$ & $41(82)$ & $25.420(2.537)$ & $9(82)$ & $22.733(2.018)$ & .002 \\
\hline Potassium (mmol/L) & $44(88)$ & $4.057(0.414)$ & $11(100)$ & $3.876(0.251)$ & .04 \\
\hline Erythrocyte sedimentation rate $(\mathrm{mm} / \mathrm{h})$ & $30(60)$ & $55.433(41.639)$ & $7(64)$ & $87.000(35.081)$ & .02 \\
\hline Neutrophils (\%) & $45(90)$ & $64.496(13.286)$ & $11(100)$ & $75.519(14.001)$ & .02 \\
\hline Lymphocytes (\%) & $45(90)$ & $25.711(10.932)$ & $11(100)$ & $17.073(9.750)$ & .01 \\
\hline Eosinophils (\%) & $45(90)$ & $1.236(1.388)$ & $11(100)$ & $0.391(1.038)$ & .009 \\
\hline Eosinophil count $\left(\times 10^{9} / \mathrm{L}\right)$ & $45(90)$ & $0.062(0.076)$ & $11(100)$ & $0.014(0.039)$ & .003 \\
\hline Lymphocyte count $\left(\times 10^{9} / \mathrm{L}\right)$ & $45(90)$ & $1.255(0.558)$ & $11(100)$ & $0.835(0.383)$ & .008 \\
\hline Packed-cell volume (hematocrit) (L/L) & $45(90)$ & $0.395(0.050)$ & $11(100)$ & $0.368(0.036)$ & .03 \\
\hline $\begin{array}{l}\text { Red blood cell distribution width-coefficient of varia- } \\
\text { tion }(\%)\end{array}$ & $45(90)$ & $12.658(1.171)$ & $11(100)$ & $12.873(0.781)$ & .03 \\
\hline
\end{tabular}

Classifiers Constructed From the FCs With Seven to Eight CLIs Could Accurately Distinguish COVID-19 From CAP

The performance of the classifiers gradually improved as the number of CLIs in the FCs increased from one to eight. However, when the number of CLIs in the FCs reached eight, the performance of the classifiers constructed by these FCs no longer significantly improved. The performance of the LR classifier algorithm constructed with the FCs with eight CLIs (ie, 8-CLI combination) was even slightly lower than those constructed by the FCs with seven CLIs (ie, 7-CLI combination). A total of 43 FCs, including five 7-CLI combinations and 38 8-CLI combinations, were determined according to the recall rate. The AUROCs of the classifiers constructed with the LR classifier, RFC, and GBC algorithms were greater than 0.85 (see Multimedia Appendix 3, Table S1). The AUROC and precision-recall curves of the classifiers constructed with the RFC, LR classifier, and GBC algorithms from the representative 7-CLI combination (ie, PCT, albumin to globulin ratio [AGR], uric acid, neutrophil count, basophil count, RBC MCV, and MCHC) showed very high performance and precision in COVID-19 prediction; their AUROCs were 1.0, 0.97, and 0.96, respectively (see Figure 2, A), and their average precision values were 1.0, 0.97, and 0.98, respectively (Figure 2, B). The AUROCs of the classifiers constructed with the RFC, LR classifier, and GBC algorithms from the representative 8-CLI combination (ie, PCT, albumin, uric acid, WBC [white blood cell] count, monocyte count, basophil count, RBC count, and MCHC) were 1.0, 0.90, and 1.0, respectively (see Figure 2, C). The AUROCs of the classifiers constructed with the three algorithms from the 7-CLI combination (ie, agr, afu, lymphocytes, neutrophil counts, eosinophil count, RBC mcv, and mchc) were $0.98,0.91$, and 0.97 , respectively (see Figure 
2, D). Feature importance results showed that basophil count was the least important in the above two representative CLI combinations, and AFU was the most important in the CLI combinations (see Figure 3). However, when basophil count was substituted with AFU in the two above-mentioned CLI combinations, the performance of the classifiers constructed with the new CLI combinations decreased (see Figure 2, E and
F). PCT and AFU were not observed to be in the same CLI combination from which an HPC could be constructed. The evidence above and the fact that only 43 FCs with seven or eight CLIs could be used to build HPCs suggested that only the FCs with specific CLIs can establish HPCs to distinguish COVID-19 from CAP.

Figure 2. Area under the receiver operating characteristic curve (AUROC) and precision-recall curve plotted for the COVID-19 vs community-acquired pneumonia (CAP) classifiers built with various feature combinations (FCs) of different clinical laboratory indicators (CLIs). At the top of each image is the CLI combination for constructing classifiers using three different classification algorithms. AFU: $\alpha$-L-fucosidase; AGR: albumin to globulin ratio; ALB: albumin; BASOC: basophil count; EOC: eosinophil count; LYM: lymphocyte; MCHC: mean corpuscular hemoglobin concentration; MCV: mean (red blood cell) corpuscular volume; MOC: monocyte count; NEUTC: neutrophil count; PCT: procalcitonin; RBC: red blood cell count; UA: uric acid; WBC: white blood cell count.
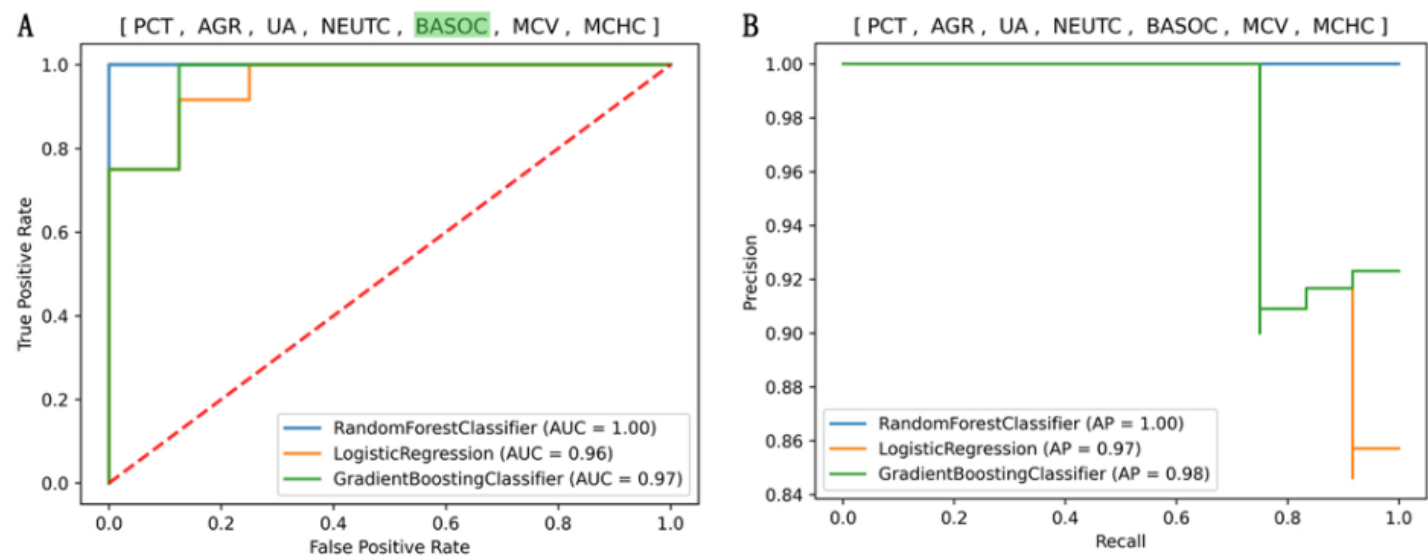

C $\quad[P C T, A L B, U A, W B C, N E U T C, B A S O C, R B C, M C H C]$
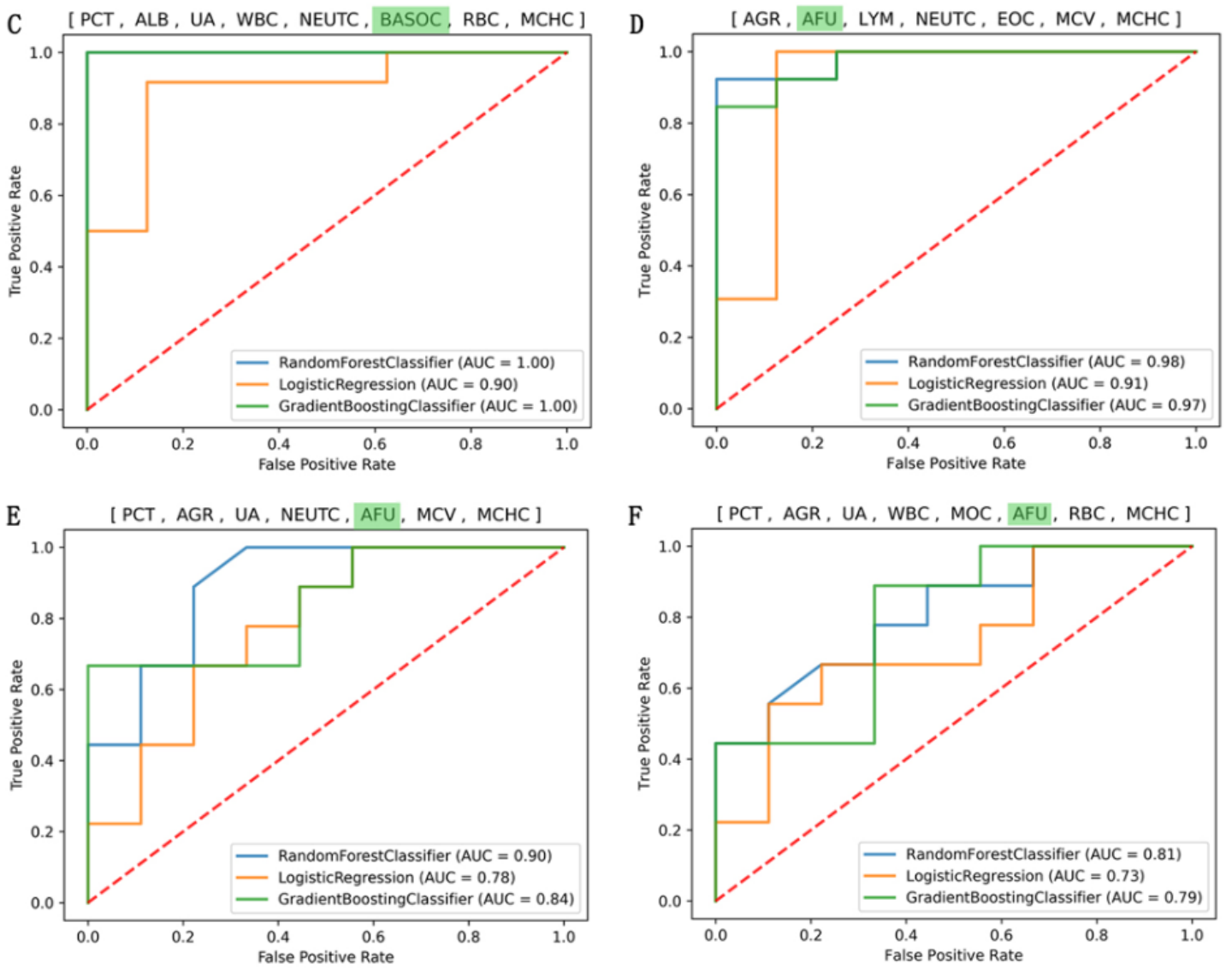

The importance of different CLIs in classifiers varied greatly, and the importance of the same CLI varied greatly among classifiers constructed by different FCs (see Figure 3). In the HPCs constructed with the 7-CLI combinations, the average

feature importance of $\mathrm{AFU}$ (26.60\%) was the highest, followed by uric acid $(25.31 \%)$ and PCT $(21.06 \%)$ (see Figure 3, A). However, in the HPCs constructed with the 8-CLI combinations, the average feature importance of uric acid (22.51\%) was the 
highest, followed by PCT (20.88\%) and MCHC (12.36\%) (see Figure 3, B). PCT and MCHC were very important to each classifier because they were included, respectively, in $100 \%$ (38/38) and 92\% (35/38) of the 8-CLI combinations (see Figure
3 , B) and in $40 \%(2 / 5)$ and $100 \%(5 / 5)$ of the 7-CLI combinations (see Figure 3, A). Uric acid was also included in all 8-CLI combinations, but its feature importance varied from $11.3 \%$ to $41.2 \%$ in different classifiers (see Figure 3, B). 
Figure 3. Usage rate and the feature importance of each clinical laboratory indicator (CLI) in the high-performance COVID-19 vs community-acquired pneumonia (CAP) classifiers. (A) The mean feature importance of each CLI in the high-performance classifiers (HPCs) constructed with the 7-CLI combinations. (B) The mean feature importance of each CLI in the HPCs constructed with the 8-CLI combinations. The histogram is represented by mean (SD). The numbers with the shadow backgrounds represent the minimum and maximum values of the feature importance of the CLI. The number indicated with the triangle symbol represents the mean feature importance of CLI in all classifiers. The number indicated with the circle represents the usage rate of the CLI in the HPC. The number in the parentheses indicates how many CLI combinations are capable of constructing the HPCs containing the CLI. AFU: $\alpha$-L-fucosidase; AGR: albumin to globulin ratio; ALB: albumin; BASOC: basophil count; EOC: eosinophil count; FC: feature combination; HGB: hemoglobin concentration; K: potassium; LYM: lymphocyte; MCHC: mean corpuscular hemoglobin concentration; MCV: mean (red blood cell) corpuscular volume; MOC: monocyte count; NEUT: neutrophil ratio; NEUTC: neutrophil count; PCT: procalcitonin; PCV: packed-cell volume (hematocrit); RBC: red blood cell count; RDW-SD: red blood cell distribution width-standard deviation; UA: uric acid; WBC: white blood cell count.
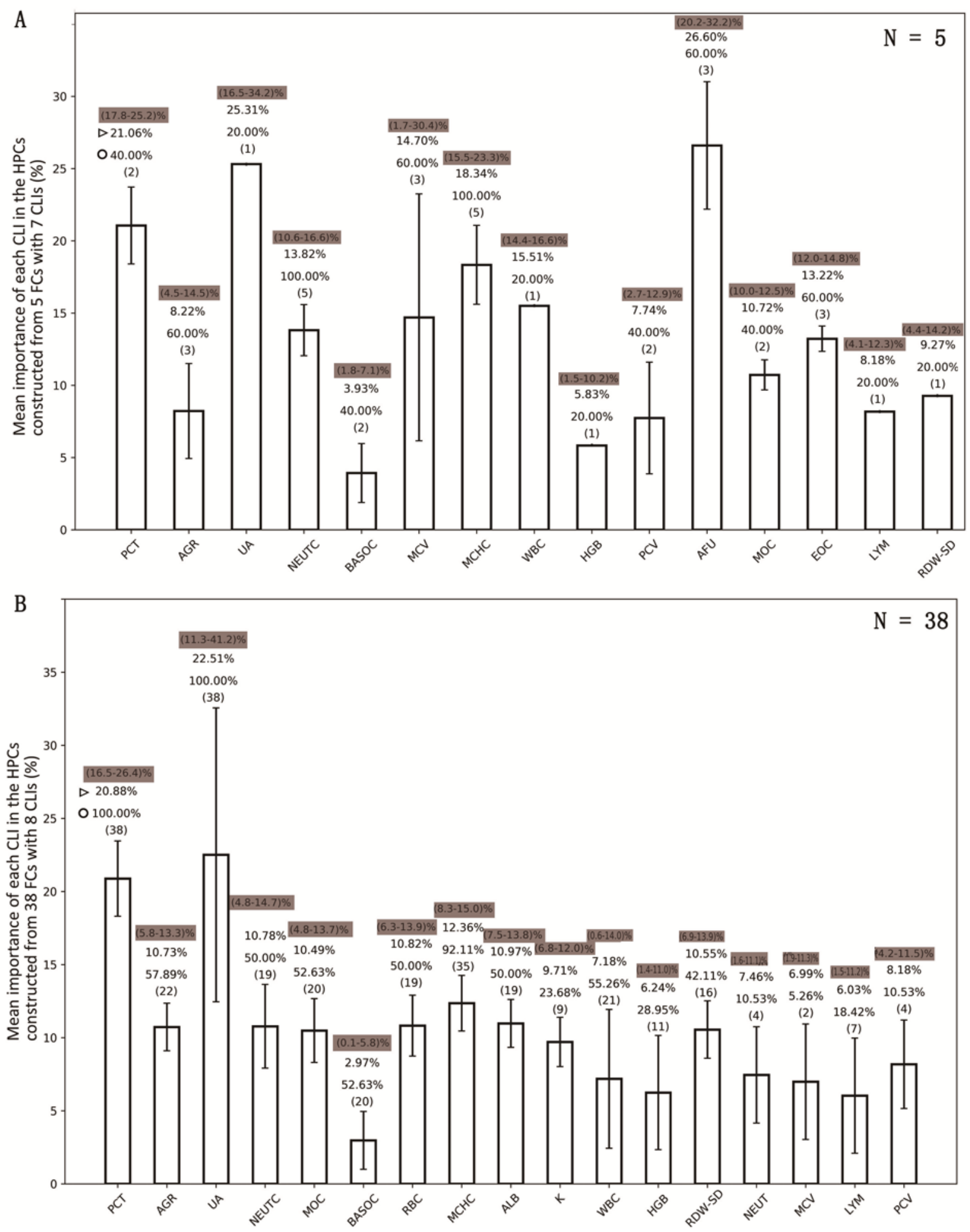


\section{Discussion}

\section{Principal Findings}

The main highlight of this study is that only a few of the common CLIs were required to establish the classifier models to accurately distinguish COVID-19 from CAP. The HPCs could only be constructed by combining several specific CLIs. Among the nearly 2 million FCs with one to eight CLIs, only $43 \mathrm{FCs}$ could be used to construct HPCs with a recall rate greater than 0.9 and an AUROC greater than 0.85 to distinguish COVID-19 from CAP.

\section{Comparison With Prior Work}

We have established many COVID-19 versus CAP HPCs with FCs consisting of only CLIs, and almost no similar research results on distinguishing COVID-19 from CAP have been reported. However, many studies have used CLIs to build ML models to help with COVID-19 diagnosis. The prediction performance of these models varied: the accuracy of these models in predicting COVID-19 was between 0.8 and 0.96 [30-32]. In addition, most of the reported ML models for the diagnosis or prediction of COVID-19 have involved more types of variables, such as CT results, clinical symptoms, and CLIs [17,32,33]. Although most of these COVID-19-related ML models were built with more than two ML algorithms, not all models constructed with each algorithm showed high performance. The methods of feature selection that were used in these studies included the recursive feature elimination algorithm [31], causal explanation models [17], and the least absolute shrinkage and selection operator regression [32]. These methods can extract the features that are closely related to the target phenotype, but whether the classifier constructed by the combination of these features has the best performance needs to be determined. The optimized FCs in this study were selected by evaluating the recall rate and AUROC for each FC with one to eight randomly selected CLIs from the differential CLIs between COVID-19 and CAP groups and by constructing classifiers using each FC with the LR classifier algorithm. The FCs that were preliminarily screened were used to build classifiers with RFC and GBC algorithms; finally, only the FCs capable of building the HPC simultaneously with the LR classifier, RFC, and GBC algorithms were selected for the final model construction.

\section{Limitations}

As reported earlier, many inflammatory factors, including IL-6 and interleukin-10 (IL-10), are closely related to COVID-19 and have diagnostic value, but neither IL-6 nor IL-10 were detected in the patients of this study. Menni et al [18] reported that loss of smell and taste is a strong predictor for COVID-19. Deviations and omissions may exist in the patients' self-reported clinical symptoms. Thus, we did not take into account the clinical symptoms when building the classifiers. The possibility that other indicators are more important in constructing COVID-19 versus CAP classifiers was not ruled out. In addition, the sample size included in this study was relatively small, and the classifiers need to be optimized with larger samples before it can be used to distinguish COVID-19 from CAP in practice.

\section{The Rationality of the Research Results}

Out of the 43 FCs, 40 contained PCT and MCHC. The feature importance of PCT in each classifier is very high, suggesting that PCT may be a good blood marker to efficiently distinguish COVID-19 from CAP. PCT is one of the markers of lower respiratory tract bacteria and other infections. The US Food and Drug Administration approved the monitoring of the beginning and the entire duration of antibiotic treatment for suspected lower respiratory tract infections based on serum PCT levels [12]. However, the elevation of serum PCT in COVID-19 patients was also reported in many studies [34]. The increase of PCT is a remarkable characteristic of patients with COVID-19 [34]. Increased serum PCT levels in both COVID-19 and CAP patients indicated that the distinction of COVID-19 from CAP could not be made simply on the basis of the increase in PCT. Compared with the normal reference values of the CLIs, the serum levels of most of the CLIs increased or decreased simultaneously in both COVID-19 and CAP patients. Thus, providing references for the diagnosis of COVID-19 or CAP directly in regard to the rise or decrease of the CLIs is difficult. However, we found that the ML classifiers constructed with the FCs with many certain CLIs could distinguish COVID-19 from CAP effectively, suggesting an advantage of ML algorithms in disease classification or diagnosis.

The COVID-19 versus CAP classifiers with the highest performance also involved PCT, MCHC, uric acid, albumin, neutrophil count, monocyte count, basophil count, RBC count, and WBC count, proposing the importance of these CLIs in differentiating COVID-19 from CAP. Few studies have reported the changing trend of MCHC in patients with COVID-19 or CAP, but the results of this study showed that MCHC decreased in both groups and was significantly lower in the CAP group than in the COVID-19 group. The reason for the decrease of MCHC may be closely related to the reduction of iron due to inflammation [35]. The IQRs of uric acid in both COVID-19 and CAP groups were within the normal reference range, but the IQR was significantly higher in the COVID-19 group than in the CAP group. Elevated uric acid is an independent risk factor of renal injury or renal dysfunction; the underlying mechanisms of uric acid elevation are very complicated [36]. The significant difference in uric acid between COVID-19 and CAP may be interpreted as follows: individuals with higher uric acid may be more susceptible to COVID-19 than those with lower uric acid levels. Uric acid exists in all 8-CLI combinations that are capable of constructing high-performance CLIs and has a high feature importance in the classifiers, suggesting that uric acid is another important marker that can distinguish COVID-19 from CAP. Zhou et al reported that albumin significantly decreased in severe and critical COVID-19 patients [37]. Serum albumin level is a good prognostic marker in CAP. A decreased albumin level is closely associated with a higher risk of mortality in patients with CAP [38]. Although albumin decreased remarkably in both COVID-19 and CAP groups, there was still a significant difference between the two groups; the decrease in the CAP group was more obvious than that in the COVID-19 group, which could contribute to the differentiation of COVID-19 from CAP. AFU contributed high feature importance in the HPCs constructed from 7-CLI combinations 
due to the significant difference in AFU between COVID-19 and CAP. An increase of serum AFU has a certain diagnostic value for primary liver cancer [39]. Thus, the higher AFU in the COVID-19 group than in the CAP group may be explained by the fact that liver injury is more common in COVID-19 than in CAP or that the diversity in AFU levels determines the difference in susceptibility to COVID-19.

\section{Recommendations}

Both PCT and AFU contributed high feature importance in the HPCs constructed from the FCs containing PCT or AFU, but the performance of the classifiers constructed from the FCs containing both PCT and AFU decreased remarkably. This result indicated that intrinsic dependence exists among some CLIs that undergo synergistic changes in individuals and can be used to construct HPCs. The internal relationship between CLIs is very complex and difficult to deconstruct. Therefore, the following method may be effective: random selection of different CLIs to construct classifiers with different classification algorithms, followed by the evaluation of the performance of each classifier, and, finally, the discovery of the FCs with certain CLIs that can be used to accurately distinguish COVID-19 from CAP.

\section{Conclusions}

The patients suffering from COVID-19 and CAP have their own characteristic profiles of CLIs, and some FCs consisting of seven or eight specific CLIs could build COVID-19 versus CAP HPCs. The usage rate and the feature importance of the CLIs in the HPCs indicated that PCT, MCHC, uric acid, albumin, AGR, neutrophil count, RBC count, monocyte count, and WBC count are the most important indicators that can distinguish COVID-19 from CAP.

\section{Acknowledgments}

We are very grateful to Associate Professor Benrong Liu of the Second Affiliated Hospital of Guangzhou Medical University for his guidance in data analysis. We are also very grateful to the doctors and nurses of Gong An County People's Hospital for their hard work to prevent as well as control COVID-19.

\section{Authors' Contributions}

XZH (huangxz020@gzucm.edu.cn) and YX (yujuanxiong@gzucm.edu.cn) share corresponding author duties for this article. XZH and YX made substantial contributions to the study concept and design. YX and PFK were in charge of drafting the manuscript. WD and WH were responsible for obtaining ethical approval, collecting data, and confirming the accuracy of the data. ZZL, QZZ, and YW performed the data analysis and interpretation. All authors agreed on the final version for submission to the journal.

\section{Conflicts of Interest}

None declared.

\section{Multimedia Appendix 1}

The statistical distribution of the plasma level of the clinical laboratory indicators (CLIs) with a significant difference between COVID19-COM (COVID-19 patient subgroup with mild and common types) and COVID19-SV (COVID-19 patient subgroup with severe and critical types). The statistical distribution was presented with a box and whisker plot. The horizontal lines within the boxes indicate the median value. The vertical lines extending below and above the boxes represent 5\%-95\% percentile values. The scale on the y-axis represents the 5th, 25th, 50th, 75th, and 95th percentile values of the CLI in the COVID19-COM subgroup. The triangles represent the upper and lower limits of the normal reference range of the laboratory index. The median of the CLI in the COVID19-SV subgroup is also represented in the y-axis. AST: aspartate aminotransferase; CO2CP: carbon dioxide binding capacity; ESR: erythrocyte sedimentation rate; $\gamma$-GGT: transglutaminase transpeptidase gamma; FIB: fibrinogen content; LDH: lactate dehydrogenase; LYM: lymphocyte; LYMPH: lymphocyte count; mCRP: micro-C-reactive protein; MYO: myoglobin; NEUT: neutrophil ratio; PA: prealbumin.

[PNG File, 403 KB-Multimedia Appendix 1]

\section{Multimedia Appendix 2}

The statistical distribution of the plasma level of the clinical laboratory indicators (CLIs) among community-acquired pneumonia (CAP), COVID19-COM (COVID-19 patient subgroup with mild and common types), and COVID19-SV (COVID-19 patient subgroup with severe and critical types). The statistical distribution was presented with a box and whisker plot. The horizontal lines within the boxes indicate the median value. The vertical lines extending below and above the boxes represent $5 \%-95 \%$ percentile values. The scale on the y-axis represents the 5th, 25th, 50th, 75th, and 95th percentile values of the CLI in the CAP group. The triangles represent the upper and lower limits of the normal reference range of the laboratory index. AFU: $\alpha$-L-fucosidase; EOC: eosinophil count; MCHC: mean corpuscular hemoglobin concentration; MCV: mean (red blood cell) corpuscular volume; MOC: monocyte count; MYO: myoglobin; RDW-SD: red blood cell distribution width-standard deviation; TT: thrombin time; UA: uric acid.

[PNG File, 204 KB-Multimedia Appendix 2] 


\section{Multimedia Appendix 3}

Clinical laboratory indicator (CLI) combinations and the hyper-parameters of the classifiers constructed by different machine learning algorithms from these CLI combinations.

[XLSX File (Microsoft Excel File), 14 KB-Multimedia Appendix 3]

\section{References}

1. Coronavirus disease (COVID-19) pandemic. World Health Organization. 2020. URL: https://www.who.int/emergencies/ diseases/novel-coronavirus-2019 [accessed 2020-05-06]

2. Coronaviridae Study Group of the International Committee on Taxonomy of Viruses. The species severe acute respiratory syndrome-related coronavirus: Classifying 2019-nCoV and naming it SARS-CoV-2. Nat Microbiol 2020 Apr;5(4):536-544 [FREE Full text] [doi: 10.1038/s41564-020-0695-z] [Medline: 32123347]

3. Ye F, Xu S, Rong Z, Xu R, Liu X, Deng P, et al. Delivery of infection from asymptomatic carriers of COVID-19 in a familial cluster. Int J Infect Dis 2020 May;94:133-138 [FREE Full text] [doi: 10.1016/j.ijid.2020.03.042] [Medline: $\underline{32247826]}$

4. Wu Z, McGoogan JM. Characteristics of and important lessons from the coronavirus disease 2019 (COVID-19) outbreak in China: Summary of a report of 72314 cases from the Chinese Center for Disease Control and Prevention. JAMA 2020 Apr 07;323(13):1239-1242. [doi: 10.1001/jama.2020.2648] [Medline: 32091533]

5. Zhang J, Tian S, Lou J, Chen Y. Familial cluster of COVID-19 infection from an asymptomatic. Crit Care 2020 Mar 27;24(1):119 [FREE Full text] [doi: 10.1186/s13054-020-2817-7] [Medline: 32220236]

6. Guan W, Ni Z, Hu Y, Liang W, Ou C, He J, China Medical Treatment Expert Group for Covid-19. Clinical characteristics of coronavirus disease 2019 in China. N Engl J Med 2020 Apr 30;382(18):1708-1720. [doi: 10.1056/NEJMoa2002032] [Medline: 32109013 ]

7. $\quad$ Li Q, Guan X, Wu P, Wang X, Zhou L, Tong Y, et al. Early transmission dynamics in Wuhan, China, of novel coronavirus-infected pneumonia. N Engl J Med 2020 Mar 26;382(13):1199-1207 [FREE Full text] [doi:

10.1056/NEJMoa2001316] [Medline: $\underline{31995857]}$

8. Linton NM, Kobayashi T, Yang Y, Hayashi K, Akhmetzhanov AR, Jung S, et al. Incubation period and other epidemiological characteristics of 2019 novel coronavirus infections with right truncation: A statistical analysis of publicly available case data. J Clin Med 2020 Feb 17;9(2):538 [FREE Full text] [doi: 10.3390/jcm9020538] [Medline: 32079150]

9. Terpos E, Ntanasis-Stathopoulos I, Elalamy I, Kastritis E, Sergentanis TN, Politou M, et al. Hematological findings and complications of COVID-19. Am J Hematol 2020 Jul;95(7):834-847 [FREE Full text] [doi: 10.1002/ajh.25829] [Medline: 32282949]

10. Ji D, Zhang D, Xu J, Chen Z, Yang T, Zhao P, et al. Prediction for progression risk in patients with COVID-19 pneumonia: The CALL score. Clin Infect Dis 2020 Sep 12;71(6):1393-1399 [FREE Full text] [doi: 10.1093/cid/ciaa414] [Medline: 32271369]

11. Wang K, Zuo P, Liu Y, Zhang M, Zhao X, Xie S, et al. Clinical and laboratory predictors of in-hospital mortality in patients with coronavirus disease-2019: A cohort study in Wuhan, China. Clin Infect Dis 2020 Nov 19;71(16):2079-2088 [FREE Full text] [doi: 10.1093/cid/ciaa538] [Medline: 32361723]

12. Kamat IS, Ramachandran V, Eswaran H, Guffey D, Musher DM. Procalcitonin to distinguish viral from bacterial pneumonia: A systematic review and meta-analysis. Clin Infect Dis 2020 Jan 16;70(3):538-542. [doi: 10.1093/cid/ciz545] [Medline: $\underline{31241140]}$

13. Robriquet L, Guery B, Fourrier F. Pulmonary coagulopathy as a new target in therapeutic studies of acute lung injury or pneumonia. Crit Care Med 2006 Sep;34(9):2510; author reply 2510-2510; author reply 2511. [doi: 10.1097/01.CCM.0000235673.40874.BD] [Medline: 16921333]

14. Schultz MJ, Haitsma JJ, Zhang H, Slutsky AS. Pulmonary coagulopathy as a new target in therapeutic studies of acute lung injury or pneumonia--A review. Crit Care Med 2006 Mar;34(3):871-877. [Medline: 16521285]

15. Ceccato A, Panagiotarakou M, Ranzani OT, Martin-Fernandez M, Almansa-Mora R, Gabarrus A, et al. Lymphocytopenia as a predictor of mortality in patients with ICU-acquired pneumonia. J Clin Med 2019 Jun 13;8(6):843 [FREE Full text] [doi: 10.3390/jcm8060843] [Medline: $\underline{31200458]}$

16. Handelman GS, Kok HK, Chandra RV, Razavi AH, Lee MJ, Asadi H. eDoctor: Machine learning and the future of medicine. J Intern Med 2018 Dec;284(6):603-619 [FREE Full text] [doi: 10.1111/joim.12822] [Medline: 30102808]

17. Schwab P, DuMont Schütte A, Dietz B, Bauer S. Clinical predictive models for COVID-19: Systematic study. J Med Internet Res 2020 Oct 06;22(10):e21439 [FREE Full text] [doi: 10.2196/21439] [Medline: 32976111]

18. Menni C, Valdes AM, Freidin MB, Sudre CH, Nguyen LH, Drew DA, et al. Real-time tracking of self-reported symptoms to predict potential COVID-19. Nat Med 2020 Jul;26(7):1037-1040 [FREE Full text] [doi: 10.1038/s41591-020-0916-2] [Medline: 32393804]

19. Ko J, Baldassano SN, Loh P, Kording K, Litt B, Issadore D. Machine learning to detect signatures of disease in liquid biopsies - A user's guide. Lab Chip 2018 Jan 30;18(3):395-405 [FREE Full text] [doi: 10.1039/c7lc00955k] [Medline: 29192299] 
20. Chinese Clinical Guidance for COVID-19 Pneumonia Diagnosis and Treatment (7th edition). Beijing, China: China National Health Commission; 2020 Mar 04. URL: http://kjfy.meetingchina.org/msite/news/show/cn/3337.html [accessed 2020-05-11]

21. Seabold S, Perktold J. Statsmodels: Econometric and statistical modeling with Python. In: Proceedings of the 9th Python in Science Conference (SciPy 2010). 2010 Jul 03 Presented at: The 9th Python in Science Conference (SciPy 2010); June 28-July 3, 2010; Austin, TX p. 92-96 URL: https://conference.scipy.org/proceedings/scipy2010/pdfs/seabold.pdf [doi: 10.25080/majora-92bf1922-011]

22. itertools - Functions creating iterators for efficient looping. The University of Auckland School of Computer Science. URL: https://www.cs.auckland.ac.nz/references/python/3.2.3-docs/library/itertools.html [accessed 2020-03-08]

23. Pedregosa F, Varoquaux G, Gramfort A, Michel V, Thirion B, Grisel O, et al. Scikit-learn: Machine learning in Python. J Mach Learn Res 2011;12:2825-2830 [FREE Full text]

24. Logistic regression, scikit-learn 0.24.1. scikit-learn. URL: https://scikit-learn.org/stable/modules/linear model. html\#logistic-regression [accessed 2020-06-12]

25. Forests of randomized trees, scikit-learn 0.24.1. scikit-learn. URL: https://scikit-learn.org/stable/modules/ensemble. html\#forests-of-randomized-trees [accessed 2020-06-12]

26. Breiman L. Random forests. Mach Learn 2001 Oct;45:5-32 [FREE Full text] [doi: 10.1023/A:1010933404324]

27. Gradient tree boosting, scikit-learn 0.24.1. scikit-learn. URL: https://scikit-learn.org/stable/modules/ensemble. html\#gradient-boosting [accessed 2020-06-12]

28. Friedman JH. 1999 Reitz Lecture. Greedy function approximation: A gradient boosting machine. Ann Stat 2001;29(5):1189-1232 [FREE Full text]

29. feature_importances_, scikit-learn 0.24.1. scikit-learn. URL: https://scikit-learn.org/stable/modules/generated/sklearn. ensemble.RandomForestClassifier.html\#sklearn.ensemble.RandomForestClassifier.feature importances [accessed 2021-02-12]

30. McDonald SA, Medford RJ, Basit MA, Diercks DB, Courtney DM. Derivation with internal validation of a multivariable predictive model to predict COVID-19 test results in emergency department patients. Acad Emerg Med 2020 Nov 28:1-9 [FREE Full text] [doi: 10.1111/acem.14182] [Medline: $\underline{33249683]}$

31. Yao H, Zhang N, Zhang R, Duan M, Xie T, Pan J, et al. Severity detection for the coronavirus disease 2019 (COVID-19) patients using a machine learning model based on the blood and urine tests. Front Cell Dev Biol 2020;8:683 [FREE Full text] [doi: 10.3389/fcell.2020.00683] [Medline: 32850809]

32. Gong J, Ou J, Qiu X, Jie Y, Chen Y, Yuan L, et al. A tool for early prediction of severe coronavirus disease 2019 (COVID-19): A multicenter study using the risk nomogram in Wuhan and Guangdong, China. Clin Infect Dis 2020 Jul 28;71(15):833-840 [FREE Full text] [doi: 10.1093/cid/ciaa443] [Medline: 32296824]

33. Schwab P, DuMont Schütte A, Dietz B, Bauer S. Clinical predictive models for COVID-19: Systematic study. J Med Internet Res 2020 Oct 06;22(10):e21439 [FREE Full text] [doi: 10.2196/21439] [Medline: 32976111]

34. Zhu Z, Cai T, Fan L, Lou K, Hua X, Huang Z, et al. Clinical value of immune-inflammatory parameters to assess the severity of coronavirus disease 2019. Int J Infect Dis 2020 Jun;95:332-339 [FREE Full text] [doi: 10.1016/j.ijid.2020.04.041] [Medline: $\underline{32334118]}$

35. Huang Y, Hu Z. Lower mean corpuscular hemoglobin concentration is associated with poorer outcomes in intensive care unit admitted patients with acute myocardial infarction. Ann Transl Med 2016 May;4(10):190 [FREE Full text] [doi: 10.21037/atm.2016.03.42] [Medline: 27294086]

36. Cao X, Wu L, Chen Z. The association between elevated serum uric acid level and an increased risk of renal function decline in a health checkup cohort in China. Int Urol Nephrol 2018 Mar;50(3):517-525. [doi: 10.1007/s11255-017-1732-6] [Medline: 29094330]

37. Zhou Y, Guo S, He Y, Zuo Q, Liu D, Xiao M, et al. COVID-19 is distinct from SARS-CoV-2-negative community-acquired pneumonia. Front Cell Infect Microbiol 2020;10:322 [FREE Full text] [doi: 10.3389/fcimb.2020.00322] [Medline: 32612961]

38. Viasus D, Garcia-Vidal C, Simonetti A, Manresa F, Dorca J, Gudiol F, et al. Prognostic value of serum albumin levels in hospitalized adults with community-acquired pneumonia. J Infect 2013 May;66(5):415-423. [doi: 10.1016/j.jinf.2012.12.007] [Medline: 23286966]

39. Deugnier Y, David V, Brissot P, Mabo P, Delamaire D, Messner M, et al. Serum alpha-L-fucosidase: A new marker for the diagnosis of primary hepatic carcinoma? Hepatology 1984;4(5):889-892. [doi: 10.1002/hep.1840040516] [Medline: $\underline{6207091]}$

\section{Abbreviations}

AFU: $\alpha$-L-fucosidase

AGR: albumin to globulin ratio

AUROC: area under the receiver operating characteristic curve

CAP: community-acquired pneumonia

CLI: clinical laboratory indicator

COVID19-COM: COVID-19 patient subgroup with mild and common types 
COVID19-SV: COVID-19 patient subgroup with severe and critical types

CRP: C-reactive protein

CT: computed tomography

FC: feature combination

GBC: gradient boosting classifier

HPC: high-performance classifier

IL-6: interleukin-6

IL-10: interleukin-10

LDH: lactate dehydrogenase

LR: logistic regression

MCHC: mean corpuscular hemoglobin concentration

mCRP: micro-C-reactive protein

MCV: mean corpuscular volume

ML: machine learning

PCT: procalcitonin

PCV: packed-cell volume

PT: prothrombin time

RBC: red blood cell

RDW-SD: red blood cell distribution width-standard deviation

RFC: random forest classifier

WBC: white blood cell

Edited by G Fagherazzi; submitted 11.08.20; peer-reviewed by J Pacheco, M Elbattah; comments to author 09.12.20; revised version received 29.12.20; accepted 01.02.21; published 22.02.21

Please cite as:

Dai W, Ke PF, Li ZZ, Zhuang QZ, Huang W, Wang Y, Xiong Y, Huang XZ

Establishing Classifiers With Clinical Laboratory Indicators to Distinguish COVID-19 From Community-Acquired Pneumonia:

Retrospective Cohort Study

J Med Internet Res 2021;23(2):e23390

URL: https://www.jmir.org/2021/2/e23390

doi: $10.2196 / 23390$

PMID: 33534722

(C)Wanfa Dai, Pei-Feng Ke, Zhen-Zhen Li, Qi-Zhen Zhuang, Wei Huang, Yi Wang, Yujuan Xiong, Xian-Zhang Huang. Originally published in the Journal of Medical Internet Research (http://www.jmir.org), 22.02.2021. This is an open-access article distributed under the terms of the Creative Commons Attribution License (https://creativecommons.org/licenses/by/4.0/), which permits unrestricted use, distribution, and reproduction in any medium, provided the original work, first published in the Journal of Medical Internet Research, is properly cited. The complete bibliographic information, a link to the original publication on http://www.jmir.org/, as well as this copyright and license information must be included. 